\title{
Conditions for Setting Exclusive Pedestrian Phases at Two-Phase Signalized Intersections considering Pedestrian-Vehicle Interaction
}

\author{
Jiawen Wang $(\mathbb{D}$, Chengcheng Yang $\mathbb{D}$, and Jing Zhao \\ Business School, University of Shanghai for Science and Technology, Shanghai 200093, China \\ Correspondence should be addressed to Jing Zhao; jing_zhao_traffic@163.com
}

Received 10 January 2020; Revised 11 December 2020; Accepted 23 December 2020; Published 6 January 2021

Academic Editor: Eleonora Papadimitriou

Copyright (C) 2021 Jiawen Wang et al. This is an open access article distributed under the Creative Commons Attribution License, which permits unrestricted use, distribution, and reproduction in any medium, provided the original work is properly cited.

\begin{abstract}
In order to analyze the effectiveness of setting exclusive pedestrian phase (EPP) under different vehicle yielding rates, the effect of EPPs on traffic efficiency is studied and a setting condition of EPP considering pedestrian-vehicle interaction is proposed in this paper. First, the main factors influencing the behavior of vehicles and pedestrians during pedestrian-vehicle interaction are analyzed, and a pedestrian-vehicle interaction (PVI) model at the crosswalk of urban road is established. Second, assuming that vehicle arrival obeys the Poisson distribution, the delay models of vehicle passengers and pedestrians crossing the street at the intersection are established, and taking the total delay of traffic participants as the main index, the setting condition of EPP are proposed. Third, based on the video of pedestrian-vehicle interaction at crosswalks, the parameters of the proposed model are calibrated. Through sensitivity analysis, the change of the total delay of traffic participants is analyzed under different conditions of pedestrian and vehicle arrival rates. Finally, by introducing pedestrian-vehicle interaction rules, a cellular automata (CA) simulation platform of pedestrian-vehicle interaction in crosswalk is established; based on the field data of Shanghai, a simulation model of intersection is established, and the total delay, queue length, and vehicle throughput under conventional signal control plan and EPP plan are compared. The results show that the pedestrian-vehicle interaction process has a great influence on the total delay of traffic participants at intersections, and pedestrian-vehicle interaction should be considered in the setting conditions of EPP. Under the same condition of vehicular flow, the more the pedestrian flow is, the smaller the delay increment will be. The higher the vehicle yielding rate is, the smaller the delay increment will be after setting EPP.
\end{abstract}

\section{Introduction}

1.1. Motivation and Contribution. At conventional twophase signalized intersections, pedestrian-vehicle interaction exists in each phase, such as vehicle yield to pedestrian and pedestrian yield to vehicle. These interaction processes lead to the increase of traffic delay for pedestrians and motor vehicles and even traffic accidents between pedestrians and vehicles when some failed interaction situation occurs, resulting in casualties and economic losses [1]. The reasonable setting of exclusive pedestrian phase (EPP) can completely eliminate the pedestrian-vehicle interaction zones at two-phase signalized intersections and ensure the safety of crossing pedestrians at the cost of slightly improving of the traffic delay [2]. In China, since the Road
Traffic Safety Law (hereinafter referred to as new traffic regulation) was amended on January 1, 2016, vehicles have to yield to pedestrians at crosswalks. However, the vehicle yielding rate had not reached $100 \%$ after the new traffic regulation was enacted. Even in the same city, yielding rates are different due to factors such as traffic flow and geometric conditions at the intersections. In addition, yielding rates vary from country to country. In developed countries (such as the United States), the enforcement of vehicle yield to pedestrian is very thorough (high and stable yielding rates), while in developing countries, it is relatively uncertain (such as China).

The new traffic regulation further ensures the pedestrian safety at crosswalks and signalized intersections and also leads to an obvious change of the pedestrian-vehicle 
interaction mechanism, which correspondingly affects the delay of the pedestrian-vehicle interaction process [3].

At present, a lot of research results of EPP have been accumulated at home and abroad and EPP optimization methods considering safety performance and traffic efficiency have been proposed. However, most of the studies on traffic efficiency take the efficiency indicators of motor vehicles or pedestrians (such as delay and traffic capacity) or the combination of the two as optimization objectives and do not fully consider traffic equity that the traffic management departments of all countries appeal [4, 5]; moreover, the great differences of the pedestrian-vehicle interaction process in different places are rarely considered in this model, which leads to certain errors in the application of the model in different cities.

In addition, due to the difference of pedestrian-vehicle interaction mechanisms, the total delay estimation methods of traffic participants are different; it is urgent to study the setting conditions of EPP under the new pedestrian-vehicle interaction mechanism. By estimating and comparing the total delay of traffic participants under different schemes, a more reasonable signal control mode at intersections can be obtained.

To fill the aforementioned two gaps, this paper first quantitatively analyzes the main factors that affect pedestrian-vehicle interaction at crosswalks and then establishes the PVI model based on the Logit model and the static game theory of incomplete information. Then, the delay models of passengers in vehicles and crossing pedestrians are established and the setting conditions of EPP are proposed with the total delay of traffic participants before and after the setting of EPP as the main index. Finally, by establishing the microsimulation platform of pedestrian-vehicle interaction based on CA and the Vissim microsimulation model of intersection, the sensitivity and application effect of EPPs in different pedestrian-vehicle interaction environments is analyzed, so as to verify the effectiveness and availability of the research results in this paper.

The contributions of this paper are as follows:

(a) Through quantitative analysis of all factors influencing the yielding rate, the main influencing factors are obtained, and based on this, the PVI model is established. In addition, the game process of the secondary stage of pedestrian-vehicle interaction is analyzed, so as to establish a pedestrian-vehicle interaction model more in line with the reality.

(b) In the setting condition of EPP, the total delay of traffic participants (convert vehicle delay into passenger delay, ped.h) is adopted as the optimization objective to reflect the equity requirements of the intersection on traffic participants.

(c) In the total delay estimation model of traffic participants, the different pedestrian-vehicle interaction situations in different cities and different intersections are considered, so as to give a more reasonable suggestion on whether to set EPPs in different pedestrian-vehicle interaction environments.

\subsection{Brief Review on EPP and Pedestrian-Vehicle Interaction} Research. An exclusive pedestrian phase (EPP) refers to the phase of signalized intersection where all vehicle approaches are displayed as red light signals, so that pedestrians can cross the intersection in all directions (including diagonal lines) at the same time [6]. Previous research on EPPs can be summarized into two categories: optimization of traffic safety and optimization of traffic efficiency [7].

In safety aspect, many studies hold that the EPP eliminates all interactions between vehicles and pedestrians, thus helping to improve the safety performance of signalized intersections [8]. In terms of traffic efficiency, the research of Tian et al. demonstrated that an EPP is beneficial in the case of large pedestrian flow and large vehicle flow; Ma et al. [7] proposed a multiobjective optimization model including EPP and traditional pedestrian phase and an algorithm to solve the optimization model. The model can determine the current optimal pedestrian phase mode according to the traffic situation and pedestrian movement of the intersection to improve the operation efficiency of the intersection. Hediyeh et al. [9] conducted a more detailed study and analyzed the pedestrian gait parameters influenced by gender, age, group size, crosswalk length, and pedestrian signal indication and understood how the pedestrian walking mechanism is affected by intersection characteristics from the micro level. Considering both efficiency and safety aspects, many studies $[2,10]$ have analyzed the setting condition of EPP, but most of them have not considered the pedestrian-vehicle interaction factor.

Different pedestrian-vehicle interaction mechanisms will affect the implementation effect of EPP [10]. Scholars at home and abroad have found that traffic environment factors $[11,12]$, pedestrian factors $[1,9,13-15]$, and vehicle factors $[12,16]$ will affect the pedestrian-vehicle interaction mechanism at signalized intersections. The pedestrian-vehicle interaction mechanism will directly affect the traffic model of signalized intersections (including capacity estimation model [17], delay estimation model [14], signal timing [18], etc.) and then change the setting condition of EPP. In the analysis aspect of pedestrian-vehicle, Kathuria and Vedagiri [19] used trajectory data to analyze the interaction behavior of unsignalized intersections in the complex traffic environment and proposed a classification method of pedestrian-vehicle interaction behavior based on the behavior of road users. Fu et al. [20] studied the safety issue of secondary pedestrian-vehicle interaction at unsignalized intersections based on the vision trajectory data. Based on previous studies, this paper analyzes the pedestrian-vehicle interaction process using intersection video data and establishes a PVI model at crosswalks as one of the input conditions of signalized intersection models.

1.3. Organization. The rest of this paper is organized as follows: the second section is problem description; in the third section, the PVI model is established; in the fourth section, the total delay estimation model of traffic participants and the EPP setting condition are proposed; the fifth and sixth sections are model validation and model analysis, which are used to analyze the effectiveness of the proposed 
EPP setting condition; and conclusions are made in the last section.

\section{Problem Description}

The total delay of traffic participants is the main index of EPP setting conditions studied in this paper. Different vehicle and pedestrian yielding rates will affect the total delay of traffic participants, thus affecting the setting condition of EPP. In order to quantify the impact of yielding rates on the delay, it is necessary to establish a model to obtain the yielding rates at intersection and then taking into account the total delay model of traffic participants before and after setting EPP.

In two-phase signalized intersections, the right-turn vehicles are usually not controlled by signal. When pedestrians are crossing, the left-turn vehicles will be released at the same time. Pedestrians have continuous interaction with turning vehicles during the green-light phase. Taking the four-approach intersection as an example, there are 3 pedestrian-vehicle interaction zones in each approach and 12 pedestrian-vehicle interaction zones in the whole interaction, as shown in Figure 1(a); after setting EPP, since pedestrians only cross during EPP time in each cycle, there is no pedestrian-vehicle interaction zone at the intersection, as shown in Figure 1(b) (conventional traffic rules are adopted in this study).

In the definition of pedestrian-vehicle interaction, Zhao et al. [21] defined that if the crossing behavior of pedestrians or vehicles changes because of the other side, it is considered that there exists pedestrian-vehicle interaction. In this paper, PVI specifically refers to when there are pedestrians waiting or crossing at the crosswalk, and the distance between vehicles and the crosswalk is about less than 50 meters [22]. At this time, vehicle will accelerate, decelerate, or stop because of the presence of pedestrians and pedestrians will correspondingly make decisions of crossing or not crossing because of the presence of vehicles. There are different types of pedestrian-vehicle interaction situations in each pedestrianvehicle interaction zone. According to the previous research $[1,23]$ and the modeling requirements of this paper, this study divides pedestrian-vehicle interaction situations into following categories: (1) vehicle yield to pedestrian and simultaneously pedestrian yield to vehicle, (2) vehicle yield to pedestrian, (3) pedestrian yield to vehicle, and (4) vehicle does not yield to pedestrian and simultaneously pedestrian does not yield to vehicle. To facilitate the model presentation, the key notations used here after are summarized in Table 1.

\section{Preliminaries: Pedestrian-Vehicle Interaction Model}

Because different yielding rates will obviously affect the setting conditions of EPP, it is necessary to establish a model to estimate the yielding rate of different intersections. In the existing studies, Yang et al. [24] has established a pedestrianvehicle interaction (PVI) model that can estimate the yielding rate at different intersections. In this study, the authors assume that the decision-making of vehicle and pedestrian is independent of each other when interactions occur. The authors use the Logit model to obtain the probability of four kinds of pedestrian-vehicle interaction (see the Problem Description section). On this basis, the evolutionary probabilities of pedestrian-vehicle interaction are obtained through the incomplete information static game theory. This paper adopts the PVI model to estimate yielding rates at intersections.

Through the aforementioned PVI model, all kinds of evolutionary situations and their corresponding probabilities during the pedestrian-vehicle interaction process can be obtained (see Table 2), which will be used as model inputs in the delay estimation of EPP setting conditions in the following section.

\section{Delay Estimation Model and the EPP Setting Condition}

Based on the PVI model established in the previous section, this section will take the total delay of traffic participants as main consideration to establish the delay estimation model of two-phase signalized intersections and give the setting condition of EPP from the perspective of traffic efficiency.

\subsection{Assumptions}

(a) In this study, saturated or oversaturated two-phase signalized intersections are not considered. For the research object in this paper, it is assumed that vehicle arrival obeys Poisson distribution (The Kolmogorov-Smirnov test method is used. The results show that the RMSE is 0.95, and the coefficient of determination and adjusted $R$-square are 09518 and 0.9490 , respectively).

(b) According to the observation video, it is found that when the pedestrian yield to vehicle, the vehicle still slows down, whereas when the vehicle yield to pedestrian, the pedestrian rarely stops (occasionally accelerating through). Accordingly, this study assumes that when the vehicle yield to pedestrian, the pedestrian does not have traffic delay, and when the vehicle yield to pedestrian, both of them have traffic delay.

(c) Considering that the intersections with EPP are mostly located in the urban core areas with large pedestrian flow, and there are often few freight vehicles. Therefore, the vehicle composition of the model established in this study includes cars and large vehicles (including buses).

4.2. Total Delay Estimation Model of Traffic Participants. In the section above, the probabilities of different pedestrian-vehicle interaction situations are obtained. In order to consider the pedestrian-vehicle interaction process in the 


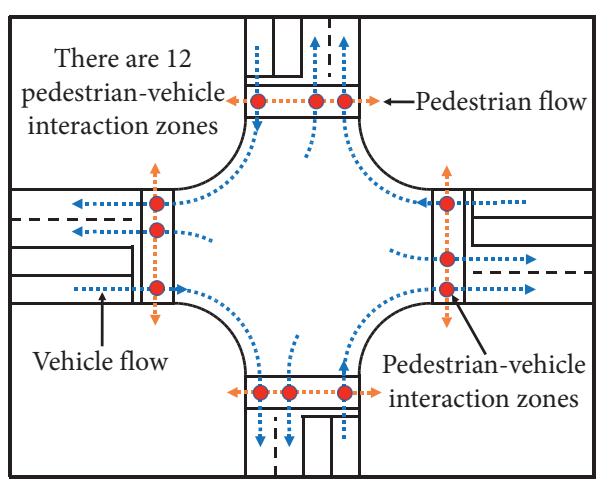

(a)

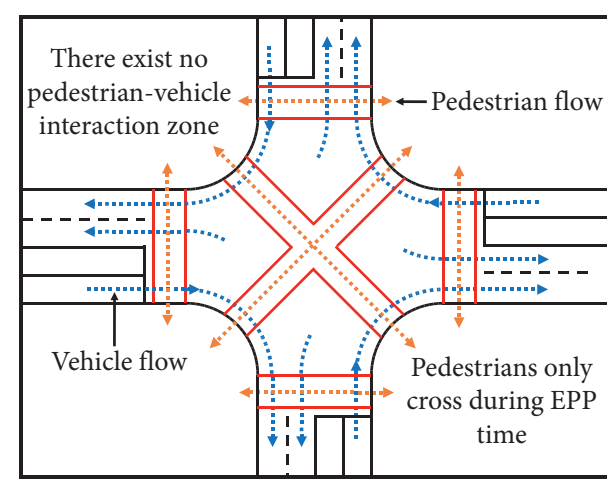

(b)

FIGURE 1: Schematic diagram of pedestrian-vehicle interaction zone before and after setting EPP: (a) before setting EPP and (b) after setting EEP.

TABLE 1: Notation and parameters.

\begin{tabular}{|c|c|}
\hline$P_{p}(i), P_{v}(i)$ & Probability of pedestrian/vehicle choosing to cross $(i=1)$ or not; $(i=2)$ \\
\hline$U_{i}^{p}, U_{i}^{v}$ & Utility function of pedestrian/vehicle \\
\hline$F_{\theta}^{p}, F_{\mu}^{v}$ & Principal component $m / n$ affecting pedestrian's/vehicle's decision-making, $\theta=1,2, \ldots, m, \mu=1,2, \ldots, n$ \\
\hline$\alpha_{\theta}^{i}, \gamma_{i}^{p}$ & Weight coefficients of pedestrian's utility function \\
\hline$\beta_{\mu}^{i}, \gamma_{i}^{v}$ & Weight coefficients of vehicle's utility function \\
\hline$x_{\delta}, y_{\delta}$ & Probability of pedestrian/vehicle choosing to cross in secondary interaction stage \\
\hline $\begin{array}{l}P_{j}, P_{k} \\
C\end{array}$ & $\begin{array}{c}\text { Vehicle/pedestrian yielding rate of } 3 \text { different pedestrian-vehicle interaction situations, } j=1,2,3, k=4,5,6 \\
\text { Cycle length, (s) }\end{array}$ \\
\hline$g$ & Effective green time, (s) \\
\hline$X$ & Saturation degree \\
\hline$T_{o}$ & The duration of observation, (h) \\
\hline$K$ & The incremental delay correction of induct \\
\hline$J$ & ental delay correction of vehicle's lane change according to upstream signal, $I=1$ at independent intersection \\
\hline$M$ & $\begin{array}{l}\text { The times of pedestrian-vehicle interaction before setting EPP during the observation time, which can be obtained by } \\
\text { modeling (obtained by linear regression in the verification case of this paper). }\end{array}$ \\
\hline
\end{tabular}

TABLE 2: Six situations of pedestrian-vehicle interaction and their corresponding probabilities.

\begin{tabular}{lcc}
\hline Six situations & Probability of each situation & Yielding rates \\
\hline Situation (2) & $P_{1}=P_{p}(1) P_{v}(2)$ \\
Situation $(1) \longrightarrow$ Situation (2) & $P_{2}=P_{p}(2) P_{v}(2) x_{1}$ \\
Situation (4) $\longrightarrow$ Situation (2) & $P_{3}=P_{p}(1) P_{v}(1) x_{2}$ \\
Situation (3) & $P_{4}=P_{v}(1) P_{p}(2)$ \\
Situation $(1) \longrightarrow$ Situation (3) & $P_{5}=P_{p}(2) P_{v}(2) y_{1}$ \\
Situation $(4) \longrightarrow$ Situation (3) & $P_{6}=P_{p}(1) P_{v}(1) y_{2}$ & $K_{p}$ \\
\hline
\end{tabular}

delay estimation model, in this paper, the ratio of the number of vehicle yield to pedestrian (include 3 situations) to the total number of pedestrian-vehicle ilnteractions is defined as the vehicle yielding rate $\left(K_{v}\right.$, see Table 2$)$, and the ratio of the number of pedestrian yield to vehicle (include 3 situations) to the total number of pedestrian-vehicle interaction is defined as the pedestrian yielding rate $\left(K_{p}\right.$, see Table 2), which will be used in the total delay estimation before setting EPP. The calculation formulas of $K_{v}, K_{p}$ are as follows:

$$
\begin{gathered}
K_{v}=\sum_{j=1}^{3} P_{j}, \\
K_{P}=\sum_{k=4}^{6} P_{k} .
\end{gathered}
$$


The total delay of traffic participants passing through the intersection consists of two parts, one is the delay of passengers in vehicle and the other is the delay of crossing pedestrians. In this paper, the total delay of traffic participants before and after setting EPP is modeled separately.

Before setting EPP, pedestrian delay includes the control delay caused by traffic signals and the delay caused by pedestrian-vehicle interaction; and vehicle delay includes the control delay caused by traffic signals and the delay caused by pedestrian-vehicle interaction.

4.2.1. Vehicle Delay. This paper adopts the delay estimation model of Highway Capacity Manual (HCM) 2010 to estimate the vehicle control delay at signalized intersections (the queue delay $d_{3}$ is 0 ):

$$
d_{\text {sig }}^{v}=\frac{0.5 C(1-(g / C))^{2}}{1-[\min (1, X)(g / C)]}+900 T_{o}\left[(X-1)+\sqrt{(X-1)^{2}+\frac{8 K I X}{C T_{o}}}\right]+d_{3} \text {. }
$$

During the pedestrian-vehicle interaction process, vehicle delay is caused by the deceleration process and the interaction process, and the interaction time is different in different situations. The vehicle delay during pedestrianvehicle interaction is given as follows:

$$
d_{\mathrm{PVI} \Phi}^{v}=\frac{V_{0}-\sqrt{V_{0}^{2}+2 a_{c} L}}{a_{c}}+t_{p v \omega}^{v}-\frac{L}{V_{0}}
$$

where $V_{0}$ is the initial velocity of the vehicle when interaction occurs, $(\mathrm{m} / \mathrm{s}) ; a_{c}$ is the acceleration when the vehicle decelerates (this item is taken as a positive value), $\left(\mathrm{m} / \mathrm{s}^{2}\right) ; L$ is the distance between deceleration position of the vehicle and the pedestrian when pedestrian-vehicle interaction occurs, $(\mathrm{m})$; and $t_{p v \omega}^{v}$ is the interaction time of vehicle in different situations, which is obtained through field investigation $(凹=p, v)$.

4.2.2. Pedestrian Delay. The control delay of pedestrian at signalized intersection $d_{\text {sig }}^{p}$ is as follows:

$$
d_{\text {sig }}^{p}=N_{p} \cdot \frac{r^{2}}{2 C}
$$

where $N_{p}$ is the pedestrian flow (ped/h) and $r$ is the time when pedestrians are not allowed to cross, $(\mathrm{s}) ; r=C-g$

During the pedestrian-vehicle interaction process, pedestrian delay is caused by the interaction process and the interaction time of different situations is different. The pedestrian delay during pedestrian-vehicle interaction is given as follows:

$$
d_{\mathrm{PVI} s}^{p}=\sum_{\omega}\left(t_{p v \omega}^{p}-\frac{d}{v_{p}}\right) M \cdot K_{\omega},
$$

where $t_{p v \omega}^{p}$ is the interaction time of pedestrian in different situations, which is obtained through field investigation $(\omega=p, v) ; d$ is the length of crosswalk; and $v_{p}$ is the crossing speed of pedestrian.

Through the delay formulas of pedestrian and vehicle and $K_{v}, K_{p}$ derived from the PVI model, the total delay $D_{1}$ of the traffic participants passing through the intersection before setting EPP can be obtained as follows:

$$
D_{1}=\left(d_{\mathrm{sig}}^{p}+\sum_{s} d_{\mathrm{PVI} s}^{p}\right) \cdot \xi+\left(d_{\mathrm{sig}}^{v} \cdot N_{c}+\sum_{\omega} d_{\mathrm{PVI} \Phi}^{v} \cdot M \cdot K_{\omega}\right) \cdot N_{v p},
$$

where $\xi$ is the pedestrian safety coefficient, which increases with the increase of the importance of pedestrians; $N_{c}$ is the vehicular flow, $(\mathrm{pcu} / \mathrm{h})$; and $N_{v p}$ is the average number of passengers carried by vehicle, (ped/pcu).

After setting EPP, there is no pedestrian-vehicle interaction zone at the intersection. The pedestrian delay only includes the control delay caused by traffic signals, and the vehicle delay includes the control delay caused by traffic signals and the delay caused by EPPs.

When an EPP is set, the pedestrian delay $d_{\text {sig }}^{p^{2}}$ is as follows:

$$
d_{\text {sig }}^{p 2}=\frac{\left(C-g_{E}\right)}{2 C} .
$$

After setting EPP, assuming that all pedestrians are passing through the intersection in the EPP time, the total delay of all traffic participants passing through the intersection $D_{2}$ is as follows:

$$
D_{2}=d_{\mathrm{sig}}^{p 2} \cdot N_{p} \cdot \xi+\left(g_{E}+d_{\mathrm{sig}}^{v}\right) \cdot N_{c} \cdot N_{v p}
$$

where $g_{E}$ is the green time of $\operatorname{EPP}(\mathrm{s})$.

4.3. Setting Condition of EPP. From the perspective of the total delay of all traffic participants passing through the intersection, the condition for setting EPPs is as follows:

$$
D_{2}<\eta \cdot D_{1} \text {, }
$$

where $\eta$ is the adjustment coefficient $(\eta \geq 1)$, considering the improvement of safety performance brought by setting EPPs, a small increase of delay should be allowed; and the value of $\eta$ should be determined according to engineering 
practice and the sensitivity analysis results of the delay model. In this paper, the determination method of $\eta$ is discussed in the Sensitivity Analysis section.

To sum up, when $D_{2} \leq \eta \cdot D_{1}$, the EPP can be set at the intersection; when $D_{2}>\eta \cdot D_{1}$, the EPP is not recommended to set.

\section{Model Validation}

In order to verify the effectiveness of the models proposed in this paper, this section will discuss three parts: first, the validity of the delay estimation model is verified; and then, the sensitivity of the EPP setting condition is analyzed to obtain the applicable conditions of EPP; and finally, the EPP setting condition are verified at the whole intersection through Vissim simulation.

5.1. Delay Model Validation. Using a CA model to simulate pedestrian traffic is an effective method [25-27]. Through comprehensive consideration of a large amount of applicability analysis and sensitivity analysis (e.g. the total simulation time reaches 3000 hours) involved in this study, after measuring the efficiency of different methods and considering the debugging of the overall model and method, a pedestrian-vehicle interaction CA model is developed in this section to conduct a detailed analysis of the proposed EPP setting conditions. The CA model can analyze the impact of more detailed changes in the pedestrian-vehicle interaction process with relatively higher efficiency (e.g. compared with VISSIM + COM method, the simulation speed increased by nearly 100 times), so it can realize more simulation analysis under more scenarios.

By introducing the mutual interference rules of pedestrians and vehicles at intersections, and coupling the NaSch model describing vehicle flow and the Blue model describing pedestrian flow, a CA simulation platform is established and the pedestrian-vehicle interaction process of an approach of a typical dual four-lane two-phase signalized intersection (right-turn lane, straight-left lane) is simulated under different vehicle yielding rates (the proportion of the number of "vehicle yield pedestrian" in the total number of pedestrianvehicle interaction).

For vehicle flow in the straight-left lane, the interaction zone is located at the crosswalk of the exit lane; for vehicle flow in the right-turn lane, the whole pedestrian-vehicle interaction process is equivalent to that the vehicles passing through two near crosswalks. Therefore, in the pedestrianvehicle interaction model of $\mathrm{CA}$, two mutually perpendicular crosswalks in the right-turn lane at intersections are regarded as two near crosswalks on a straight lane. The schematic diagram of the CA pedestrian-vehicle interaction model is shown in Figure 2.

In the process of establishing the CA simulation platform, this paper refers to the modeling ideas and relevant parameters of the previous researchers [25, 28, 29] based on CA to study pedestrians and vehicles. The parameters include geometric parameters of road (lane width, crosswalk length, and width), vehicle regularity parameters, pedestrian area parameters, and speed parameters. On this basis, according to the characteristics of the research object in this paper, the maximum speed of vehicles in different functional lanes and different positions are reset accordingly. The parameter calibration process is provided in the below sections.

5.2. Calibration of the Vehicle CA Model. Model parameters are mainly divided into two categories: one is the external parameters of the model, including intersection geometry parameters and pedestrian signal parameters; and the other is the internal parameters of the model, mainly including vehicle speed-related parameters at different positions of the crosswalk. Tables 3 and 4 classify the parameters involved in the model. The value of external parameters is mainly set according to the specific conditions of the signalized intersection, which has great flexibility. The internal parameters are relatively fixed and determined by empirical values or iterative methods. The parameter calibration process of the CA model is carried out as shown in Figure 3. Table 5 shows the comparison of measured and simulated vehicle capacity values.

The location of model parameter calibration and verification in this paper is the right-turn lane and the straightleft lane at the south entrance of the intersection of Anbo Road-Shagang Road, Yangpu District, Shanghai, and the calibration and verification process are observed independently. The average vehicle capacity of the above two lanes is $1103 \mathrm{pcu} / \mathrm{h}$ and $487 \mathrm{pcu} / \mathrm{h}$ respectively. The relative deviation between the simulation result and the actual result is set as $10 \%$. The relative deviation is calculated as follows:

$$
\varepsilon_{1}=\left|\frac{\overline{C^{s}}-\overline{C^{r}}}{\overline{C^{r}}}\right| \times 100 \%,
$$

where $\varepsilon$ is the relative deviation; $C^{s}$ is the vehicle capacity obtained by simulation; and $C^{r}$ is the vehicle capacity obtained from the actual survey. The internal parameters of the CA model are calibrated according to the steps shown in Figure 3, and the final results of the internal parameters are shown in Table 3. The relative deviation between the simulation and actual value of the right-turn lane is $7.89 \%$, and the relative deviation between the simulation and actual value of the straight-left lane is $5.00 \%$.

5.3. Calibration of the Pedestrian CA Model. The calibration and verification of pedestrian CA model parameters are conducted in two crosswalks at the south entrance of the intersection of Anbo Road and Shagang Road in Yangpu District, Shanghai, where the calibration and verification processes are observed independently. The average pedestrian crossing time is taken as the index value. The average crossing time of the above two crosswalks (converted into a single lane) is $4.18 \mathrm{~s}$ and $3.92 \mathrm{~s}$, respectively. At the same time, the unit time step of simulation time should be converted. According to the survey, the average crossing speed of pedestrians on the above two crosswalks is $0.97 \mathrm{~m} / \mathrm{s}$ and $1.04 \mathrm{~m} / \mathrm{s}$, respectively, so when the simulation is carried 


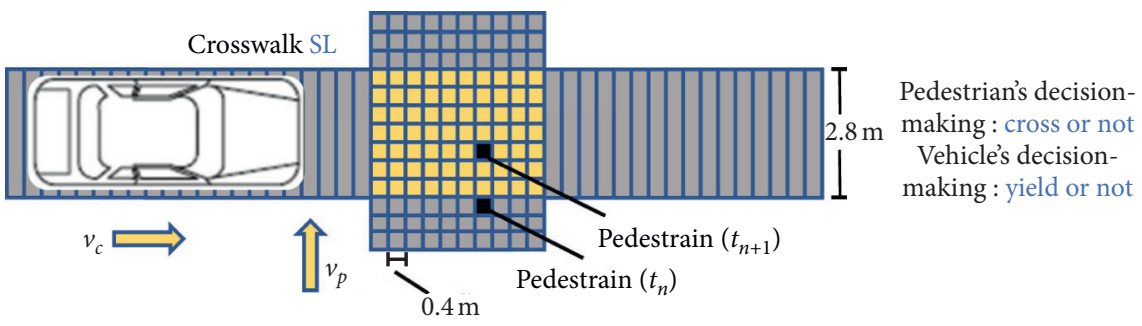

(a)

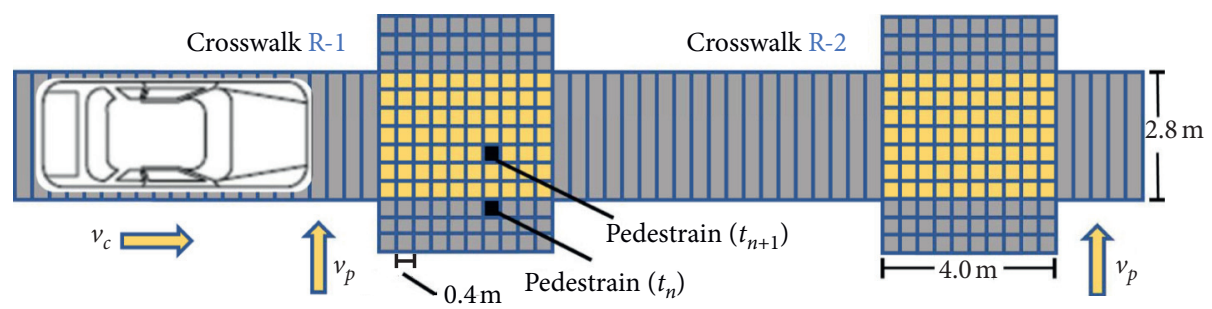

(b)

FIGURE 2: Schematic diagram of the cellular automata model for vehicle and pedestrian.

TABLE 3: Vehicle CA model parameters and calibration results.

\begin{tabular}{lccc}
\hline $\begin{array}{l}\text { Parameter } \\
\text { classification }\end{array}$ & Parameters & Notation & Value \\
\hline \multirow{4}{*}{$\begin{array}{c}\text { Crosswalk length } \\
\text { Crosswalk width } \\
\text { Width of right-turn } \\
\text { lane }\end{array}$} & $W_{1}$ & 7 \\
& $W_{2}$ & 7 \\
& $\begin{array}{c}\text { Width of straight-left } \\
\text { lane }\end{array}$ & $W_{3}$ & 7 \\
& Vehicle length & $V_{l}$ & 15 \\
Internal parameters & Vehicle width & $V_{w}$ & 7 \\
\hline & Maximum speed 1 & $v_{\max 1}^{v}$ & 40 \\
& Maximum speed 2 & $v_{\max 2}^{v}$ & 32 \\
& Maximum speed 3 & $v_{\max 3}^{v}$ & 20 \\
\hline
\end{tabular}

out on these two crosswalks, the unit time step is equal to $0.4 / 0.97=0.41 \mathrm{~s}$ and $0.4 / 1.04=0.38 \mathrm{~s}$, respectively. According to this conversion relationship, the pedestrian signal time, pedestrian arrival, and relevant parameters are converted into simulation unit for input. The relative deviation between the simulation results and the actual results is set to be $10 \%$. The relative deviation is calculated using formula (11). Table 6 shows the comparison of measured and simulated pedestrian crossing time, and the relative deviation between the simulation results and the actual results in the two scenarios is $9.81 \%$ and $6.63 \%$, respectively.

$$
\varepsilon_{2}=\left|\frac{\overline{\mathrm{CT}^{s}}-\overline{\mathrm{CT}^{r}}}{\overline{\mathrm{CT}^{r}}}\right| \times 100 \% .
$$

In this simulation, crosswalks are divided into square cell grids, each cell can only accommodate one person, and the cell width of the right-turn lane is equal to the side length of the cell of the crosswalk. Pedestrians and vehicles choose whether to pass or not according to the given probabilities in the model when they enter the interaction zone. Parameters
TABle 4: Pedestrian CA model parameters and calibration results.

\begin{tabular}{lccc}
\hline Parameter classification & Parameters & Notation & Value \\
\hline \multirow{2}{*}{ External parameters } & Pedestrian length & $P_{l}$ & 1 \\
& Pedestrian width & $P_{w}$ & 1 \\
\multirow{2}{*}{ Internal parameters } & Pedestrian speed 1 & $v_{1}^{p}$ & 2 \\
& Pedestrian speed 2 & $v_{2}^{p}$ & 3 \\
\hline
\end{tabular}

of the experimental lanes in the microsimulation environment are shown in Table 7.

In practice, the yielding rate can be estimated for a given intersection [24]. The simulation is carried out in the case of vehicle yielding rates of $0.75,0.50$, and 0.25 , respectively, and 10 times simulation are conducted for each yielding rate before and after setting EPP, respectively. The errors between CA simulation and delay model are shown in Table 8. After calculation, the average errors before and after setting EPP are $8.13 \%, 7.57 \%, 8.57 \%, 6.91 \%, 8.09 \%$, and $7.72 \%$, respectively, under the three yielding rates. The average error of the two methods is less than $10 \%$, which proves the validity of the proposed delay model.

\section{Model Analysis}

6.1. Sensitivity Analysis. On the basis of verifying the validity of the PVI model and delay model, in this part, the sensitivity of EPP setting conditions is analyzed through the CA simulation platform established above and total traffic efficiency and total throughput of the two modes are compared. The simulation is carried out in the case of vehicle yielding rates of $0.75,0.50$, and 0.25 , respectively. The comparison of total delay (s) and total throughput (ped/h) of traffic participants before and after setting EPP in the simulation intersection is shown in Figures 4 and 5, respectively. Parameters of the 


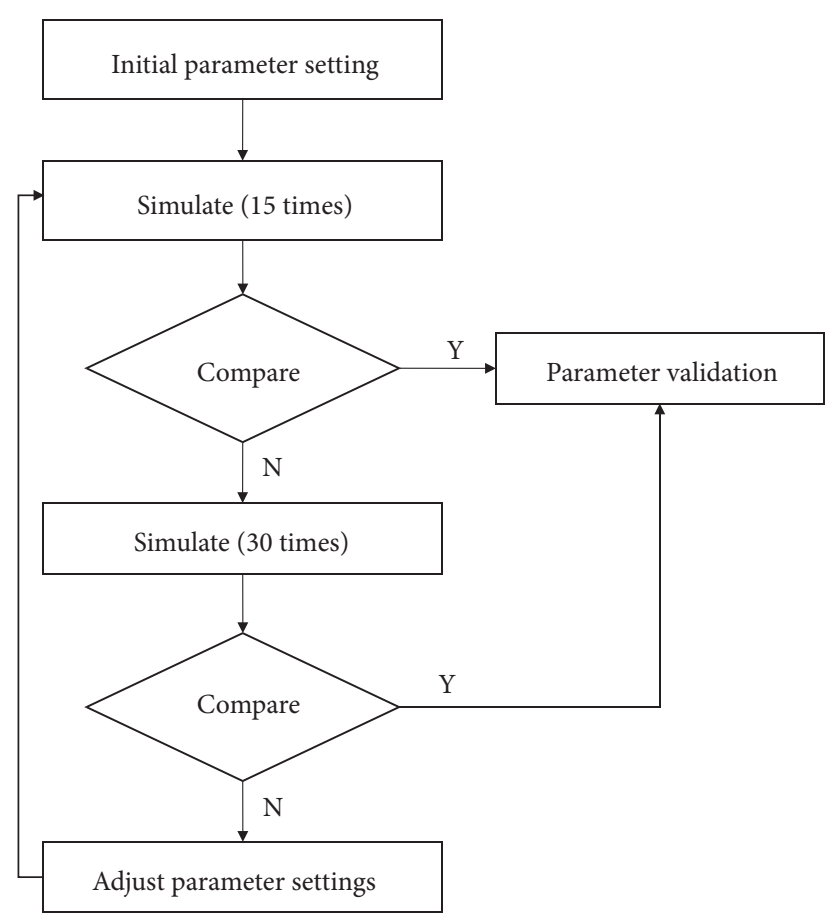

FIgURE 3: Calibration process of CA model parameters.

TABLE 5: Comparison of measured and simulated vehicle capacity values.

\begin{tabular}{|c|c|c|c|c|c|c|c|c|c|c|c|c|c|c|c|}
\hline & G1 & G2 & G3 & G4 & G5 & G6 & G7 & G8 & G9 & G10 & G11 & G12 & G13 & G14 & G15 \\
\hline $\mathrm{Me}$ & 032 & 056 & 59 & 136 & 039 & 1259 & 119 & 1046 & 1036 & 1055 & 1063 & 124 & 142 & 129 & 1069 \\
\hline & 893 & 926 & 1026 & 047 & 1040 & 1106 & 1056 & 1178 & 9 & 943 & 98 & & 1069 & 32 & 919 \\
\hline & 452 & 433 & 521 & 503 & 459 & 498 & 487 & 502 & 5 & 49 & 5 & & & 501 & 448 \\
\hline Simulation value $(\mathrm{pcu} / \mathrm{h})$ & 389 & 462 & 457 & 446 & 482 & 426 & 456 & 423 & 448 & 521 & 456 & 511 & 434 & 552 & 479 \\
\hline
\end{tabular}

TABLE 6: Comparison of measured and simulated pedestrian crossing time.

\begin{tabular}{|c|c|c|c|c|c|c|c|c|c|c|c|c|c|c|c|}
\hline & G1 & G2 & G3 & $\mathrm{G} 4$ & G5 & G6 & G7 & G8 & G9 & G10 & G11 & G12 & G13 & G14 & G15 \\
\hline $\mathrm{Me}$ & 66 & 2 & .36 & 3.45 & 2.91 & .38 & 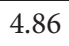 & 4.92 & 5.24 & 3.95 & J & 2. & 4.55 & 74 & 4.20 \\
\hline Sin & 75 & 5.16 & 01 & 2.2 & & 6 & 4.7 & 3 & 5.3 & 4.06 & 8 & 3.26 & & & 4.95 \\
\hline Mea & 3.95 & 4.6 & 3.69 & 6.5 & 3.8 & 3.86 & 3. & 4.95 & 3.46 & 2. & 4. & 2.66 & 3. & 2.68 & 4.05 \\
\hline Simulation value (s) & 3.16 & 4.82 & 3.07 & 7.34 & 3.15 & 4.22 & 3.77 & 3.75 & 2.86 & 2.96 & 3.57 & 2.85 & 3.16 & 2.9 & 3.27 \\
\hline
\end{tabular}

experimental lanes in the microsimulation environment are shown in Table 7.

In Figure 4, the red and black lines are the dividing lines where the total delay increment before and after setting EPP is 0 and $20 \%$, respectively, that is, the delay increment in area $i-1$ after setting EPP is less than 0; the range of delay increment in area $i-2$ is $0-20 \%$; and the delay increment in area $i-3$ is more than $20 \%$ (see Table 9). From the sensitivity analysis, this study draws the following conclusions:

(a) In same vehicular flow, the more the pedestrian flow is, the smaller the delay increment is after setting EPP and the better the setting condition is.

(b) The higher the vehicle yielding rate is, the smaller the delay increment is. It shows that the yielding rate has a great influence on the total delay of traffic participants at intersections. It is important to take yielding rate as a model variable in this study.

In addition, Figure 4 shows the case when $\eta$ is $1.00-1.20$. It can be seen that under different vehicle yielding rates, the larger the $\eta$ value is, the larger the flow range of the recommended EPP will be expanded. Based on the analysis results of this study, in practical engineering applications, the value of $\eta$ is suggested to be $1.15-1.20$ at the intersection of high-density areas such as commercial district, which emphasizes the safety of pedestrians. The value of $\eta$ at the intersection of residential area and leisure area is suggested to be 1.05-1.15, which considers both efficiency and safety. 
TABLE 7: Parameter setting table of simulation.

\begin{tabular}{lcc}
\hline Parameters & Straight-left lane & Right-turn lane \\
\hline Vehicle yielding rate & $99 \%$ & $99 \%$ \\
Lane width (m) & 3 & 3 \\
Gradient (degree) & 0 & 0 \\
Base saturation flow (pcu/h) & 1800 & 1500 \\
Cycle length C (s) & 90 & 90 \\
Effective green time $g(\mathrm{~s})$ & 42 & 42 \\
Correction parameter of signal linkage PF & 1.0 & 1.0 \\
Incremental delay correction of actuated control $K$ & 0.5 & 0.5 \\
Correction of upstream signal delay I & 1.0 & 1.0 \\
Average car load factor (ped/pcu) & 1.5 & 1.5 \\
Average bus load factor (ped/pcu) & 25 & 25 \\
Proportion of buses & $7 \%$ & $7 \%$ \\
Ratio of turning traffic & $60 \%$ & $60 \%$ \\
The length of EPP $(\mathrm{s})$ & 26 & 26 \\
Observation time $T$ (h) & 1 & 1 \\
\hline
\end{tabular}

TABLE 8: Comparison between CA simulation and delay model.

\begin{tabular}{lccccccccccc}
\hline Vehicle yielding rate & Error & 1 & 2 & 3 & 4 & 5 & 6 & 7 & 8 & 9 & 10 \\
\hline \multirow{2}{*}{0.75} & Before (\%) & 7.44 & 6.44 & 4.57 & 6.78 & 6.24 & 7.42 & 11.78 & 9.50 & 10.93 & 10.26 \\
& After (\%) & 12.62 & 5.64 & 6.36 & 12.18 & 9.37 & 3.20 & 4.15 & 8.39 & 4.46 & 9.30 \\
0.50 & Before (\%) & 5.98 & 7.87 & 9.10 & 10.67 & 5.13 & 4.93 & 13.15 & 13.56 & 7.43 & 7.88 \\
& After (\%) & 9.84 & 8.09 & 3.53 & 7.41 & 12.88 & 8.24 & 2.30 & 7.77 & 1.34 & 7.72 \\
0.25 & Before (\%) & 7.80 & 11.27 & 10.07 & 5.85 & 7.27 & 5.89 & 8.42 & 4.74 & 8.17 & 11.46 \\
& After (\%) & 8.09 & 3.21 & 5.85 & 10.80 & 7.51 & 12.27 & 14.34 & 3.94 & 3.89 & 7.37 \\
\hline
\end{tabular}

At the intersection of industrial zone and suburb, the value is suggested to be 1.00-1.05, which emphasizes traffic efficiency.

Figure 5 shows the comparison of total throughput of traffic participants before and after setting EPP. In area $i-1$, except for a small area where the throughput decreased significantly, the throughput decreased slightly or slightly increased in most areas; in area $i-2$, when there are both more or less pedestrians and vehicles arriving, the throughput before and after the setting changes little, while the central area changes greatly; in area $i-3$, the throughput of almost the whole area decreased significantly after setting EPP (see Table 9). The analysis of the change of total throughput shows that the EPP setting conditions with total delay as the main indicator can reduce the total delay of intersections at the expense of relatively less total throughput.

6.2. Case Study. In order to verify the effectiveness of the EPP setting condition proposed in this paper under the simulation environment close to the actual traffic environment, a Vissim simulation model is established to simulate the situation where all vehicles yield to pedestrians (vehicle yielding rate, 100\%).

Taking the typical dual four-lane two-phase signalized intersection shown above as an example, the whole intersection is simulated under two different scenarios. Because a pedestrian can cross street diagonally after setting EPP, the pedestrian input flows are set different before and after setting EPP. The setting of simulation parameters is shown in Table 10, and the flow input in each direction is shown in Table 11.

In the established Vissim network, the east-west secondary road gives way to the north-south main road and vehicles yield to pedestrians. For each simulation scenario, 5 different random seeds were selected for 5 simulation experiments to reduce the random influence on the results. Simulations were conducted in the above two scenarios, and the results are shown in Table 12.

In the case that the vehicle yielding rate is $100 \%$, after setting EPP at the intersection of scenario 1, the delay of passengers in vehicles increases, the queue length of vehicles increases, the delay of pedestrians decreases, and the total delay of traffic participants at the intersection increases. Therefore, it is unreasonable to set EPP at the intersection of scenario 1.

After setting EPP at the intersection of scenario 2, the delay of passengers in vehicles increases, the throughput of vehicles increases, the delay of pedestrians decreases, the throughput of pedestrians increases, and the total delay of traffic participants decreases. In this scenario, the total delay is reduced by about $4 \%$, which is consistent with the results of the sensitivity analysis, indicating that setting EPP in scenario 2 is reasonable and proving the validity of the model of EPP setting conditions proposed in this study.

In addition, compared with the low vehicle yielding rates, when the vehicle yielding rate is relatively high, the total delay of traffic participants decreases more after setting EPP from which it can be seen how the vehicle yielding rate affects EPP setting conditions. 


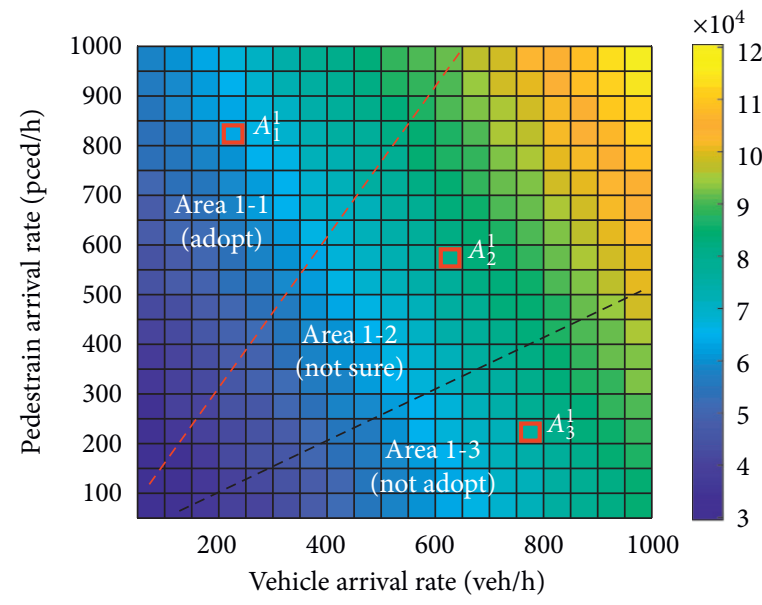

(a)

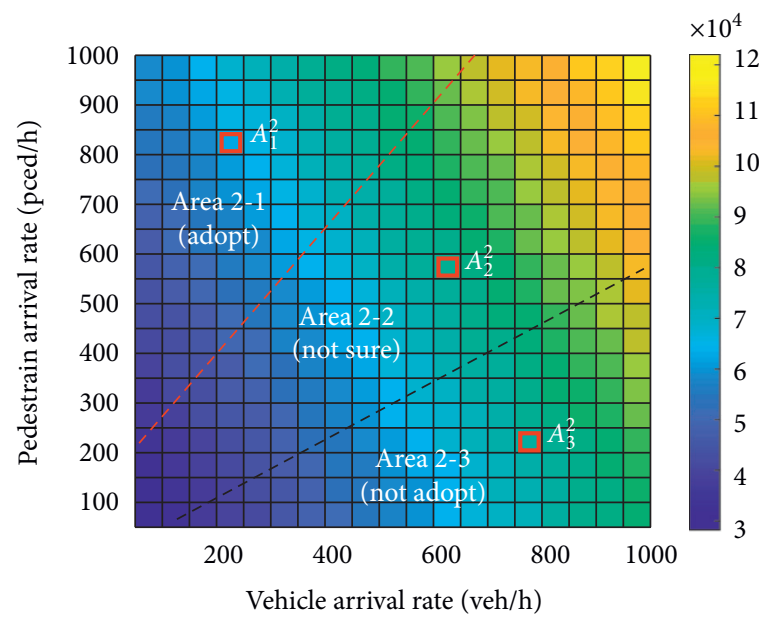

(c)

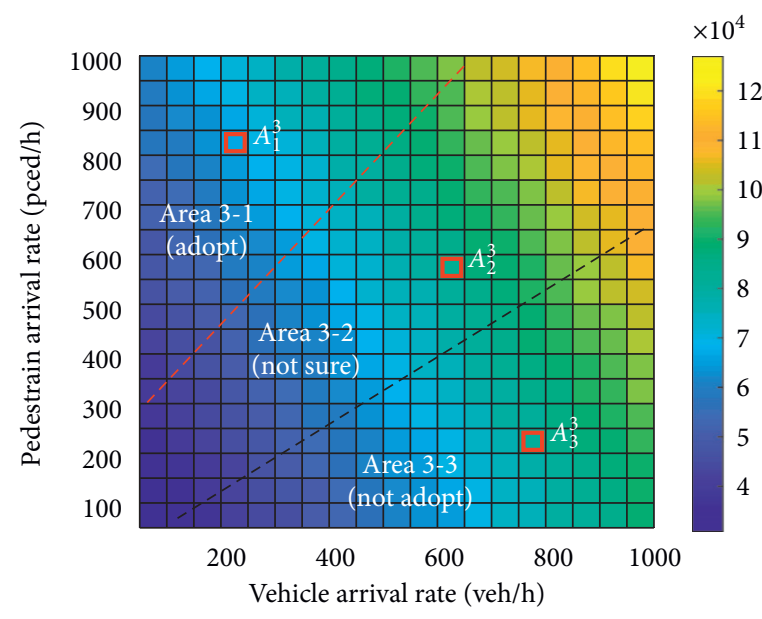

(e)

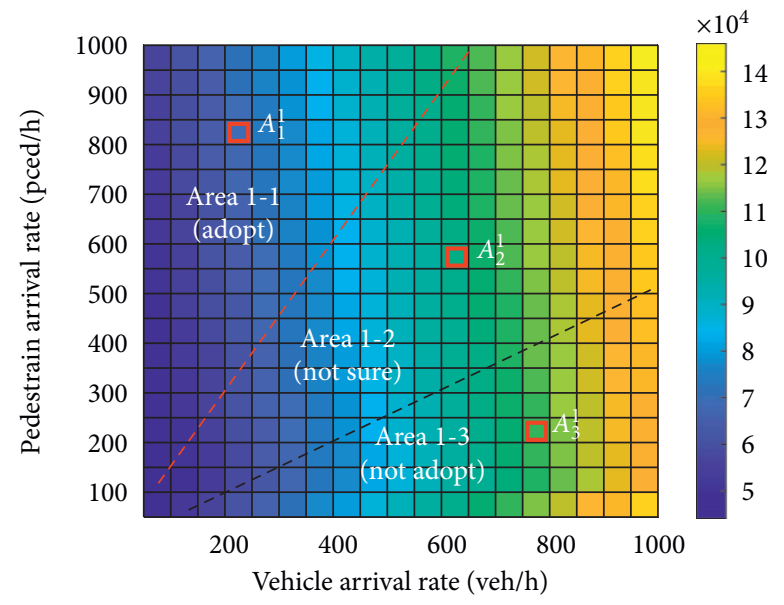

(b)

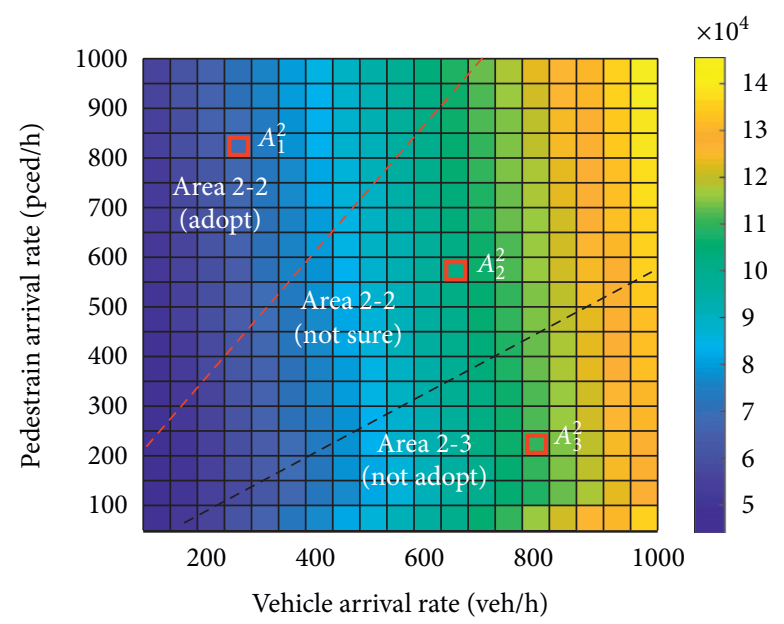

(d)

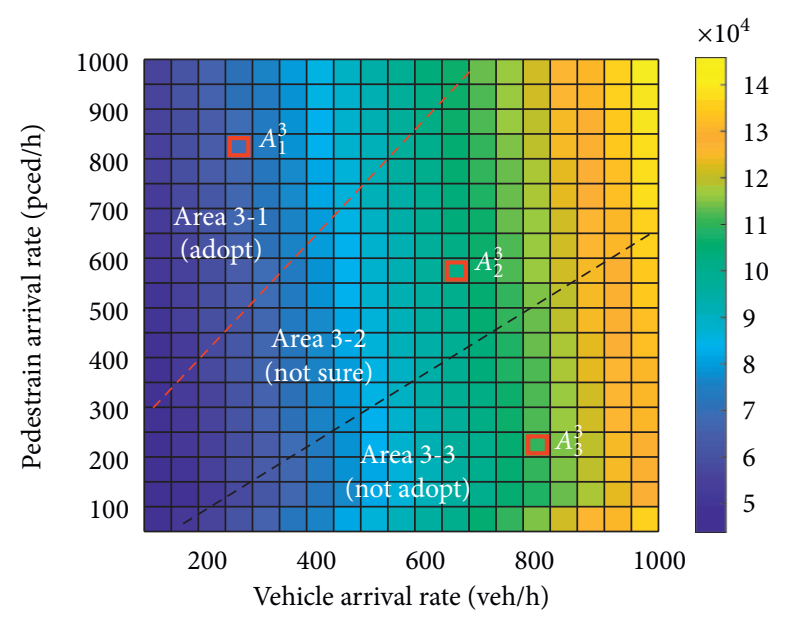

(f)

FIGURE 4: Total delay of traffic participants under different vehicle yielding rates: (a) before setting EPP (vehicle yielding rate, $75 \%$ ), (b) after setting EPP (vehicle yielding rate, 75\%), (c) before setting EPP (vehicle yielding rate, 50\%), (d) after setting EPP (vehicle yielding rate, 50\%), (e) before setting EPP (vehicle yielding rate, 25\%), and (f) after setting EPP (vehicle yielding rate, 25\%). 


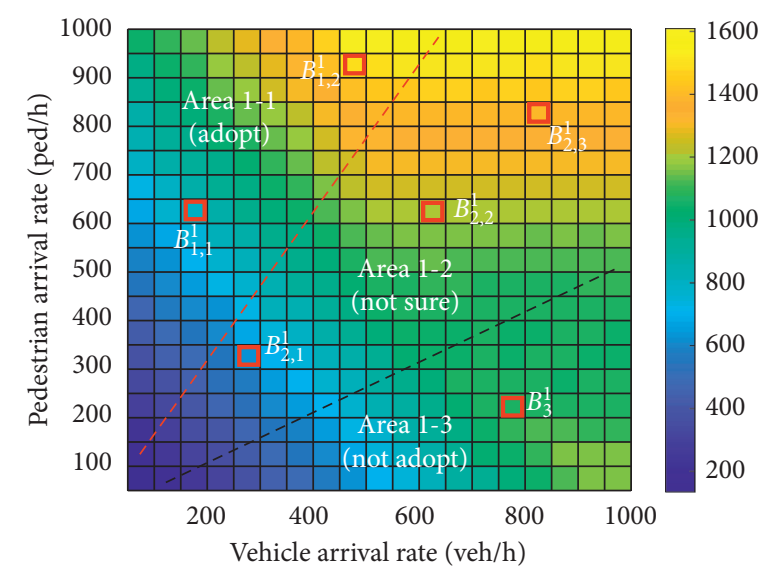

(a)

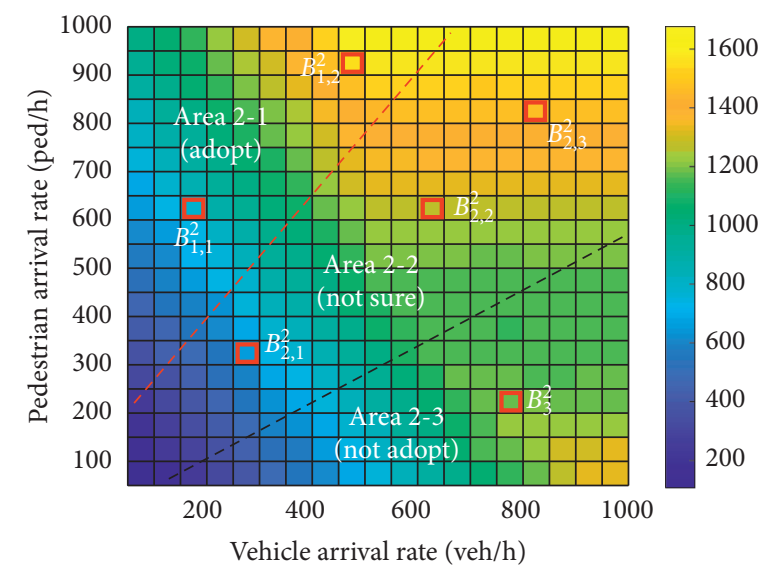

(c)

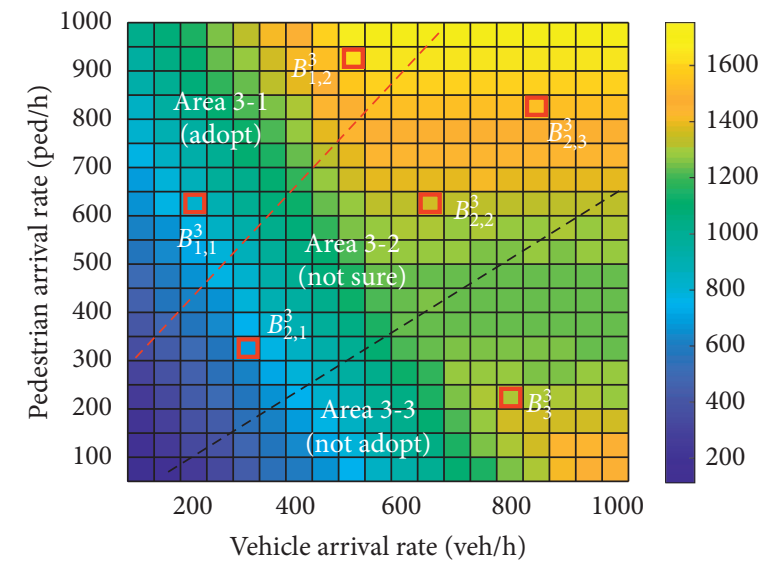

(e)

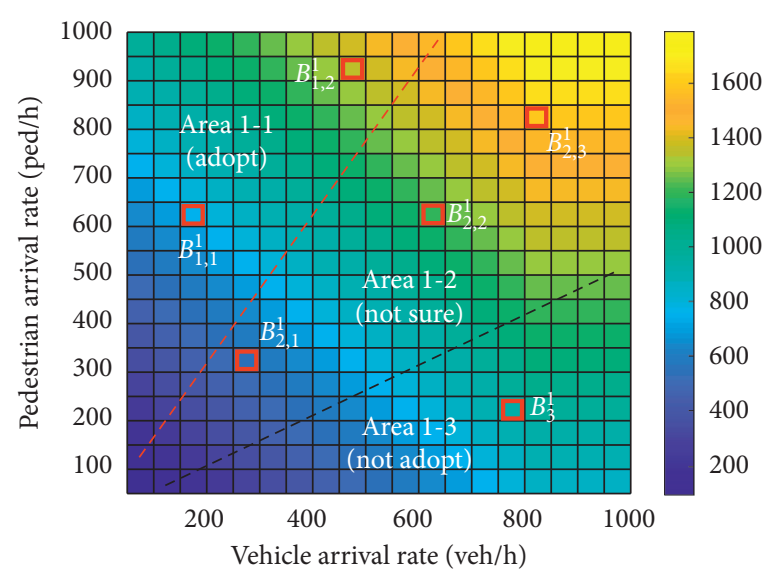

(b)

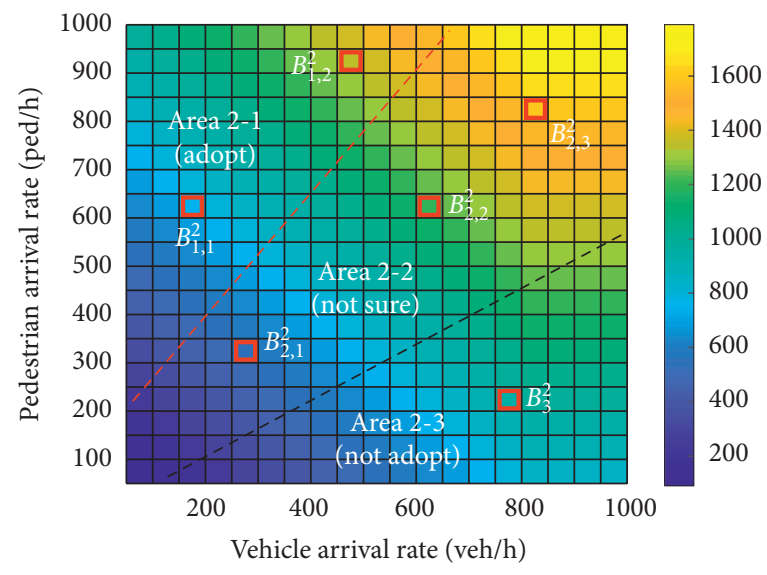

(d)

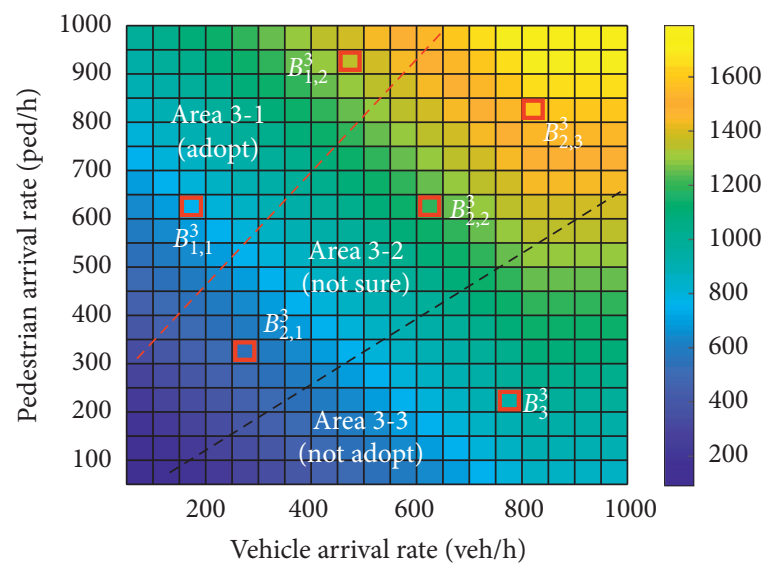

(f)

FIGURE 5: Total throughput of traffic participants under different vehicle yielding rates: (a) before setting EPP (vehicle yielding rate, 75\%), (b) after setting EPP (vehicle yielding rate, 75\%), (c) before setting EPP (vehicle yielding rate, 50\%), (d) after setting EPP (vehicle yielding rate, 50\%), (e) before setting EPP (vehicle yielding rate, 25\%), and (f) after setting EPP (vehicle yielding rate, 25\%). 
TABLE 9: Comparison of total delay and throughput before and after setting EPP (representative points).

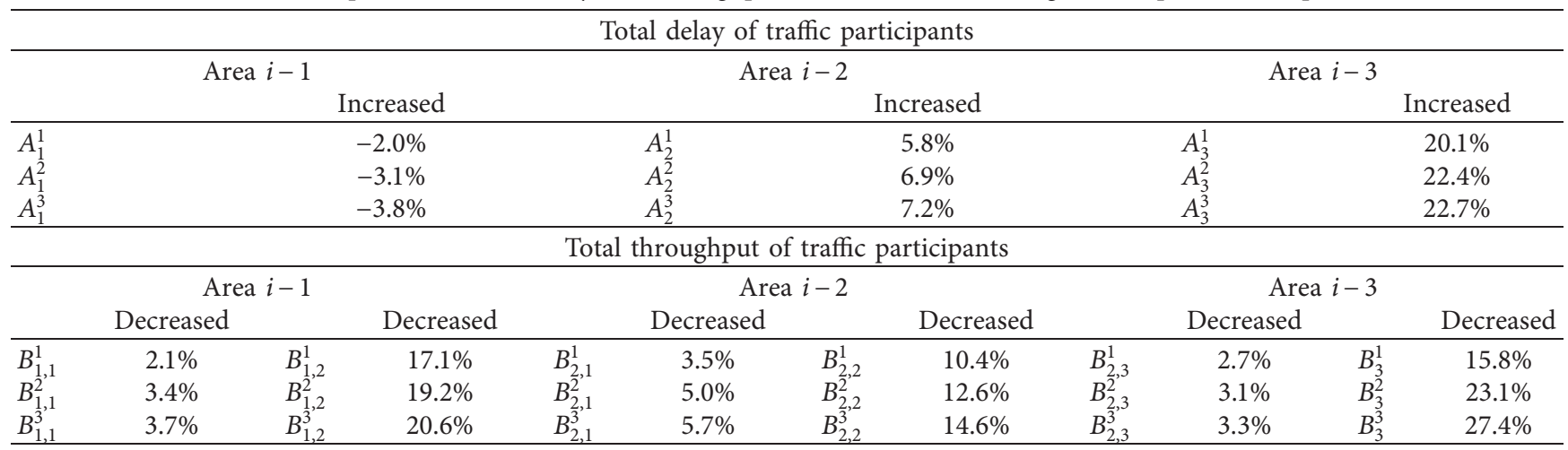

TABle 10: Parameters in microscopic simulation.

\begin{tabular}{|c|c|c|c|c|c|c|c|}
\hline \multicolumn{2}{|l|}{ Parameter category } & \multicolumn{2}{|c|}{ Crosswalk SL } & \multicolumn{2}{|c|}{ Crosswalk R-1 } & \multicolumn{2}{|c|}{ Crosswalk R-2 } \\
\hline \multicolumn{2}{|l|}{ Traffic environment parameters } & \multicolumn{2}{|c|}{$\begin{array}{l}\text { Cycle length } C(\mathrm{~s}) \\
90\end{array}$} & \multicolumn{4}{|c|}{$\begin{array}{l}\text { Effective green time } g(\mathrm{~s}) \\
42\end{array}$} \\
\hline \multirow{5}{*}{ Arrival rates of pedestrians and vehicles } & Case 1 & $\begin{array}{l}q_{v}(\mathrm{veh} / \mathrm{h}) \\
q_{p}(\mathrm{ped} / \mathrm{h})\end{array}$ & $\begin{array}{l}0-1000 \\
0-1000\end{array}$ & $\begin{array}{l}q_{v}(\mathrm{veh} / \mathrm{h}) \\
q_{p}(\mathrm{ped} / \mathrm{h})\end{array}$ & $\begin{array}{l}0-1000 \\
0-1000\end{array}$ & $\begin{array}{l}q_{v}(\mathrm{veh} / \mathrm{h}) \\
q_{p}(\mathrm{ped} / \mathrm{h})\end{array}$ & $\begin{array}{l}0-1000 \\
0-1000\end{array}$ \\
\hline & \multirow{2}{*}{ Case 2} & $q_{v}(\operatorname{veh} / \mathrm{h})$ & $0-1000$ & $q_{v}(\mathrm{veh} / \mathrm{h})$ & $0-1000$ & $q_{v}(\mathrm{veh} / \mathrm{h})$ & $0-1000$ \\
\hline & & $q_{p}(\mathrm{ped} / \mathrm{h})$ & $0-1000$ & $q_{p}(\mathrm{ped} / \mathrm{h})$ & $0-1000$ & $q_{p}(\mathrm{ped} / \mathrm{h})$ & $0-1000$ \\
\hline & \multirow{2}{*}{ Case 3} & $q_{v}(\mathrm{veh} / \mathrm{h})$ & $0-1000$ & $q_{v}(\mathrm{veh} / \mathrm{h})$ & $0-1000$ & $q_{v}(\mathrm{veh} / \mathrm{h})$ & $0-1000$ \\
\hline & & $q_{p}(\mathrm{ped} / \mathrm{h})$ & $0-1000$ & $q_{p}(\mathrm{ped} / \mathrm{h})$ & $0-1000$ & $q_{p}(\mathrm{ped} / \mathrm{h})$ & $0-1000$ \\
\hline \multirow{6}{*}{ Yielding rates of pedestrians and vehicles } & \multirow{2}{*}{ Case 1} & $K_{v}$ & 0.75 & $K_{v}$ & 0.75 & $K_{v}$ & 0.75 \\
\hline & & $K_{p}$ & 0.25 & $K_{p}$ & 0.25 & $K_{p}$ & 0.25 \\
\hline & \multirow{2}{*}{ Case 2} & $K_{v}^{P}$ & 0.50 & $K_{v}^{P}$ & 0.50 & $K_{v}^{P}$ & 0.50 \\
\hline & & $K_{p}$ & 0.50 & $K_{p}$ & 0.50 & $K_{p}$ & 0.50 \\
\hline & \multirow{2}{*}{ Case 3} & $K_{v}^{p}$ & 0.25 & $K_{v}^{p}$ & 0.25 & $K_{v}^{p}$ & 0.25 \\
\hline & & $K_{p}$ & 0.75 & $K_{p}$ & 0.75 & $K_{p}$ & 0.75 \\
\hline
\end{tabular}

TABLE 11: Metrix of flow input.

\begin{tabular}{lcc}
\hline Traffic flow & Case 1 & Case 2 \\
\hline Left-turn vehicular flow $(\mathrm{pcu} / \mathrm{h})$ & 200 & 100 \\
Straight line vehicular flow $(\mathrm{pcu} / \mathrm{h})$ & 150 & 75 \\
Right-turn vehicular flow $(\mathrm{pcu} / \mathrm{h})$ & 150 & 75 \\
Pedestrian flow after setting EPP (ped/h) & 83 & 166 \\
Pedestrian flow before setting EPP $(\mathrm{ped} / \mathrm{h})$ & 125 & 250 \\
\hline
\end{tabular}

TABLE 12: Simulation results of the two cases.

\begin{tabular}{lcccc}
\hline \multirow{2}{*}{ Evaluating indicators } & \multicolumn{2}{c}{ Case 1 } & \multicolumn{2}{c}{ Case 2 } \\
& Before setting EPP & After setting EPP & Before setting EPP & After setting EPP \\
\hline Passenger delay in vehicle (h) & 111.3 & 132.1 & 41.3 & 49.4 \\
Average queue length (m) & 34.4 & 21.7 & 4.35 & 7.16 \\
Vehicle throughput (pcu) & 1890 & 1954 & 1017 & 1021 \\
Pedestrian delay (h) & 19.5 & 11.6 & 25.9 & 15.4 \\
Pedestrian throughput (ped) & 1306 & 1272 & 2604 & 2607 \\
Total delay (h) & 125.8 & 143.7 & 67.2 & 64.8 \\
\hline
\end{tabular}




\section{Conclusions}

This paper studied the pedestrian-vehicle interaction process at two-phase signalized intersections and puts forward the concept of yielding rate; a PVI model at crosswalks in urban road is established, and on this basis, the total delay estimation model of traffic participants considering the yielding rate is established; and then taking the total delay before and after setting EPP of traffic participants as main indexes, the setting condition of EPP is given. The analysis results of this study show that when the vehicle flow is constant, the higher the pedestrian flow is, the better the effect of setting EPP is, and the yielding rate has an effect on the setting condition of EPP; thus, it is of great importance to consider the yielding rate as an important factor in the setting condition of EPP. Finally, a CA simulation platform of pedestrian-vehicle interaction and a Vissim simulation model are established, through which the effectiveness of the proposed EPP setting condition is verified.

In addition to the total delay of traffic participants considered in this paper, capacity and safety performance of intersections are also important factors affecting the setting condition of EPP. In the future, based on the video data of EPP intersections, the determination method of the adjustment coefficient will be further studied, and the setting condition of EPP considering safety and efficiency comprehensively will be given. On the other hand, in the verification of EPP setting conditions, this paper adopts the method of establishing a CA model of pedestrian-vehicle interaction because the method has a high operation efficiency under the premise of guaranteed analysis results. However, other verification methods have better advantages (e.g., the VISSIM + COM interface can also realize the pedestrian-vehicle interaction process with different probabilities, which has higher model credibility). Therefore, future research work will further compare the differences between CA methods and other methods in the simulation of pedestrian-vehicle interaction and then find a more suitable method to explore the impact of pedestrian-vehicle interaction on the setting conditions of EPP at intersections.

\section{Data Availability}

This paper cited data from YANG's paper [24] on the impact of crossing pedestrians on right-turn capacity. Other data used to support the findings of this study are included within the paper.

\section{Conflicts of Interest}

The authors declare that they have no conflicts of interest.

\section{Acknowledgments}

This work was financially supported by the Shanghai Sailing Program (Grant no. 19YF1435100).

\section{References}

[1] X. Xin, N. Jia, S. Ma, and J. Mu, "Empirical and simulation study of traffic delay at un-signalized crosswalks due to conflicts between pedestrians and vehicles," Transportmetrica B: Transport Dynamics, vol. 7, no. 1, pp. 637-656, 2019.

[2] W. Ma, D. Liao, Y. Liu, and H. K. Lo, "Optimization of pedestrian phase patterns and signal timings for isolated intersection," Transportation Research Part C: Emerging Technologies, vol. 58, pp. 502-514, 2015.

[3] T. Fu, L. Miranda-Moreno, and N. Saunier, "A novel framework to evaluate pedestrian safety at non-signalized locations," Accident Analysis \& Prevention, vol. 111, pp. 23-33, 2018.

[4] Q. I. Ahmed, H. Lu, and S. Ye, "Urban transportation and equity: a case study of Beijing and Karachi," Transportation Research Part A: Policy and Practice, vol. 42, no. 1, pp. 125-139, 2008.

[5] G. Lefebvre-Ropars, C. Morency, P. A. Singleton, and K. J. Clifton, "Spatial transferability assessment of a composite walkability index: the pedestrian index of the environment (PIE)," Transportation Research Part D: Transport and Environment, vol. 57, pp. 378-391, 2017.

[6] L. Kattan, S. Acharjee, and R. Tay, "Pedestrian scramble operations," Transportation Research Record: Journal of the Transportation Research Board, vol. 2140, no. 1, pp. 79-84, 2009.

[7] W. Ma, Y. Liu, and K. L. Head, "Optimization of pedestrian phase patterns at signalized intersections: a multi-objective approach," Journal of Advanced Transportation, vol. 48, no. 8, pp. 1138-1152, 2014.

[8] J. N. Ivan, K. McKernan, Y. Zhang, N. Ravishanker, and S. A. Mamun, "A study of pedestrian compliance with traffic signals for exclusive and concurrent phasing," Accident Analysis \& Prevention, vol. 98, pp. 157-166, 2017.

[9] H. Hediyeh, T. Sayed, and M. H. Zaki, "The use of gait parameters to evaluate pedestrian behavior at scramble phase signalized intersections," Journal of Advanced Transportation, vol. 49, no. 4, pp. 523-534, 2015.

[10] Y. Zhang, S. A. Mamun, J. N. Ivan, N. Ravishanker, and K. Haque, "Safety effects of exclusive and concurrent signal phasing for pedestrian crossing," Accident Analysis \& Prevention, vol. 83, pp. 26-36, 2015.

[11] R. Van Houten, J. E. L. Malenfant, R. Blomberg, B. Huitema, and J. Hochmuth, "High-visibility enforcement on driver compliance with pedestrian right-of-way laws: 4-year followup," Transportation Research Record: Journal of the Transportation Research Board, vol. 2660, no. 1, pp. 58-65, 2017.

[12] S. Stapleton, T. Kirsch, T. J. Gates, and P. T. Savolainen, "Factors affecting driver yielding compliance at uncontrolled midblock crosswalks on low-speed roadways," Transportation Research Record: Journal of the Transportation Research Board, vol. 2661, no. 1, pp. 95-102, 2017.

[13] T. Goddard, K. B. Kahn, and A. Adkins, "Racial bias in driver yielding behavior at crosswalks," Transportation Research Part F: Traffic Psychology and Behaviour, vol. 33, pp. 1-6, 2015.

[14] Y. Zheng and L. Elefteriadou, "A model of pedestrian delay at unsignalized intersections in urban networks," Transportation Research Part B: Methodological, vol. 100, pp. 138-155, 2017.

[15] E. A. Bourquin, R. W. Emerson, D. Sauerburger, and J. M. Barlow, "Conditions that influence drivers' behaviors at roundabouts: increasing yielding for pedestrians Who are visually impaired," Journal of Visual Impairment \& Blindness, vol. 112, no. 1, pp. 61-71, 2018.

[16] M. Sucha, D. Dostal, and R. Risser, "Pedestrian-driver communication and decision strategies at marked crossings," Accident Analysis \& Prevention, vol. 102, pp. 41-50, 2017. 
[17] D. Wei, H. Xu, W. Kumfer, H. Liu, and Z. Wang, "Vehicular traffic capacity at unsignalized crosswalks with probabilistic yielding behavior," Transportation Research Record: Journal of the Transportation Research Board, vol. 2483, no. 1, pp. 80-90, 2015.

[18] L. Zhang, J. Zhu, and B. Shuai, "Multi-objective optimization of signal timing at congestion intersection by considering pedestrian behavior," Computer Engineering and Applications, vol. 2015, no. 16, pp. 223-227, 2015.

[19] A. Kathuria and P. Vedagiri, "Evaluating pedestrian vehicle interaction dynamics at un-signalized intersections: a proactive approach for safety analysis," Accident Analysis \& Prevention, vol. 134, p. 105316, 2020.

[20] T. Fu, W. Hu, L. Miranda-Moreno, and N. Saunier, "Investigating secondary pedestrian-vehicle interactions at nonsignalized intersections using vision-based trajectory data," Transportation Research Part C: Emerging Technologies, vol. 105, pp. 222-240, 2019.

[21] J. Zhao, J. O. Malenje, J. Wu, and R. Ma, "Modeling the interaction between vehicle yielding and pedestrian crossing behavior at unsignalized midblock crosswalks," Transportation Research Part F: Traffic Psychology and Behaviour, vol. 73, pp. 222-235, 2020.

[22] L.-Y. Wei, Y.-F. Cui, and D.-Y. Li, "Evolution mechanism of conflict between pedestrian and vehicle based on evolutionary game theory," Acta Physica Sinica, vol. 67, no. 19, Article ID 190201, 2018.

[23] W. Van Haperen, S. Daniels, T. De Ceunynck, N. Saunier, T. Brijs, and G. Wets, "Yielding behavior and traffic conflicts at cyclist crossing facilities on channelized right-turn lanes," Transportation Research Part F: Traffic Psychology and Behaviour, vol. 55, pp. 272-281, 2018.

[24] C. Yang, J. Wang, and J. Dong, "Capacity model of exclusive right-turn lane at signalized intersection considering pedestrian-vehicle interaction," Journal of Advanced Transportation, vol. 2020, Article ID 1534564, 19 pages, 2020.

[25] V. J. Blue and J. L. Adler, "Cellular automata microsimulation for modeling bi-directional pedestrian walkways," Transportation Research Part B: Methodological, vol. 35, no. 3, pp. 293-312, 2001.

[26] L. Lu, C.-Y. Chan, J. Wang, and W. Wang, "A study of pedestrian group behaviors in crowd evacuation based on an extended floor field cellular automaton model," Transportation Research Part C: Emerging Technologies, vol. 81, pp. 317-329, 2017.

[27] J. Zhao, V. L. Knoop, and M. Wang, "Two-dimensional vehicular movement modelling at intersections based on optimal control," Transportation Research Part B: Methodological, vol. 138, pp. 1-22, 2020.

[28] J. B. Sun Ze and X. Li, "The study of the interference between pedestrians and vehicles based on cellular automation model," Acta Physica Sinica, vol. 61, no. 10, 2012.

[29] J. Yang, W. Deng, J. Wang, Q. Li, and Z. Wang, "Modeling pedestrians' road crossing behavior in traffic system microsimulation in China," Transportation Research Part A: Policy and Practice, vol. 40, no. 3, pp. 280-290, 2006. 